EXEMPLARIa Classica

Journal of Classical Philology

14, 2010, pp. $227-248$

ISSN 1699-3225

\title{
LA TRADICIÓN MANUSCRITA DEL BREVIARIUM DE JOHANNES DE SANCTO PAULO ${ }^{1}$
}

\author{
Ana Isabel Martín Ferreira \\ Universidad de Valladolid \\ anabel@fyl.uva.es \\ Alejandro García GonZÁleZ \\ Universidad de Valladolid \\ jandrogarciagonzalez@gmail.com
}

Summary

This article compiles the manuscript tradition of the Breviarium de signis, causis et curis morborum. This is an unpublished latin medical text, written by Johannes de Sancto Paulo in the twelfth century. This author is linked to the so-called Salerno Medical School. The testimonies of the codices provide data, hitherto unknown, about this author and his work.

KEYWORDS

Middle Ages, Medical School of Salerno, Breviarium, Johannes de Sancto Paulo, manuscript tradition

\section{RESUMEN}

Este artículo recopila la tradición manuscrita del Breviarium de signis, causis et curis morborum. Se trata un texto latino médico inédito, compuesto en el siglo XII por Johannes de Sancto Paulo, un autor vinculado a la llamada Escuela Médica Salernitana. Los testimonios de los códices aportan datos, hasta ahora desconocidos, sobre el autor y su obra.

Palabras Clave

Edad Media, Escuela Médica Salernitana, Breviarium, Johannes de Sancto Paulo, tradición manuscrita.

Fecha de recepción: 30/06/2010

Fecha de aceptación y versión final: 21/09/2010

La edición crítica de textos medievales de contenido científico y médico según los criterios de la crítica textual moderna es un campo de investigación en el que aún queda un inmenso trabajo por realizar. Los esfuerzos llevados a cabo hasta hoy para dar a conocer este tipo de literatura han comenzado a

${ }^{1}$ Este trabajo ha sido realizado en el seno del proyecto de investigación FFI2008-00618/ FILO, y dentro del Programa de Movilidad de los Recursos Humanos de Investigación "Ayudas de estancias de movilidad postdoctoral en el extranjero, incluidas becas MEC/FULBRIGHT y Cátedra Príncipe de Asturias" (Ref. 2008-0196), ambos financiados por el Ministerio Español de Ciencia e Innovación. 
ofrecer sus primeros frutos ${ }^{2}$, pero cuantitativamente son pocas las obras que han pasado por este proceso y todavía muchas a las que sólo tenemos acceso a través de ediciones renacentistas, las cuales tienen la misma validez que cualquier otro testimonio manuscrito de las obras. En el caso de la literatura médico-botánica adscrita a la llamada Escuela Médica de Salerno contamos además con la colección de textos publicados en el siglo XIX por Salvatore De Renzi en su obra Collectio Salernitana ${ }^{3}$, pero indudablemente los criterios de edición fueron otros y los textos allí publicados no conforman el total de la literatura médica de origen salernitano. Por ello en ocasiones nos encontramos ante obras que no sólo no fueron incluidas por De Renzi en su colección, sino que ni siquiera conocieron tampoco edición alguna en época renacentista, lo que convierte a sus autores en auténticos desconocidos y a estos textos en prácticamente inaccesibles. Es el caso del personaje y la obra que nos ocupan, Johannes de Sancto Paulo, autor salernitano de, entre otras obras, un Breviarium de signis, causis et curis morborum hasta ahora inédito.

\section{Johannes de Sancto Paulo}

Poco sabemos con certeza de este escritor médico vinculado a la escuela médica salernitana ${ }^{4}$, calificado por De Renzi de 'personaggio misterioso' e identificado con el compañero de estudios al que Gilles de Corbeil dedicaba el poético apelativo de Castalius 5 . Valentin Rose, en su edición del Viaticus de este último autor ${ }^{6}$, apuntaba a su identificación con cierto cardenal y obis-

${ }^{2}$ Este es uno de los principales objetivos del Grupo de Investigación Speculum medicinae de la Universidad de Valladolid (España), que dirige el Prof. Enrique Montero Cartelle. Este grupo ha editado, entre otros, textos médicos de Constantino el Africano, Arnaldo de Villanova, Bernardo de Gordon o textos adscritos a la Escuela Médica de Salerno como el glosario Alphita o el poema Flos medicinae.

${ }^{3}$ Collectio Salernitana: ossia documenti inediti, e trattati di medicina appartenenti alla Scuola Medica Salernitana, a cura di Salvatore De Renzi, 5 vols., Napoli, FiliatreSebezio, 1852-9.

${ }^{4}$ Cf. L. Gaffuri, "Giovanni di San Paolo", Dizionario biografico degli italiani, vol. 56, Roma 2001, 212-7; M. H. Green, "Johannes de Sancto Paulo”, Medieval Science, Technology and Medicine. An Encyclopedia, ed. Th. Glick-S J. Livese-F. Wallis, New York-London 2005, 286; Johanns von Sancto Paulo, 'Liber de simplicium medicinarum virtutibus' und ein anderer Salernitaner Traktat, 'Quae medicinae pro quibus morbis donandae sunt' nach dem Breslauer Codex herausgegeben..., ed. G. H. Krömer, Borna-Leipzig 1920; Flores diaetarum: eine salernitanische Nahrungsmitteldiätetik aus dem XII. Jahrhundert, ed. H. J. Ostermuth, Borna-Leipzig 1919; A. Paravicini Bagliani, Medicina e scienze della natura alla corte dei papi nel Duecento, Spoleto 1991, 72-3; R. Sharpe, Titulus. Identifying Medieval latin Texts. An Evidence-Based Approach, Turnhout 2003, 136-41; K. Sudhoff, "Salerno, Montpellier und Paris um 1200”, Archiv für Geschichte der Medizin 20, 1928, 51-62 (esp. 52-3 y 56-7). 330.

${ }^{5}$ S. De Renzi (ed.), Collectio Salernitana 4 (1856), 612; cf. también 1 (1852), 245 y 3 (1854),

${ }^{6}$ Egidii Corboliensis Viaticus. De signis et symptomatibus aegritudinum, ed. V. Rose, Leipzig 1907. 
po de la Sabina, en la Italia central. A esto se suma el dato descubierto por Karl Sudhoff en el códice 178 de la Biblioteca de Pommersfeld, en el que el comentarista del De urinis de Egidio alude a Johannes de Sancto Paulo como cardinali et episcopo albano, es decir, prelado de Albi, a orillas del Tarn, en el Sureste de Francia, no lejos de Montpellier. Según estos datos, se le ha vinculado también a la Escuela Montepesulana. Sin embargo, todo ello aparece siempre vinculado a documentación eclesiástica, posterior al siglo XIII, en la que no es infrecuente encontrar entre diferentes cargos eclesiásticos el nombre de 'Johannes'. En esta línea, tampoco se ha verificado la hipótesis según la cual acabó siendo monje benedictino (¿abad?) en el monasterio de San Pablo de Roma, en el que habría muerto no más allá de 1214-1215. Pero, si algo nos interesa desde el punto de vista de la composición de sus obras, es el dato de que se trata de un personaje que, como muchos otros, alcanzó prebendas eclesiásticas gracias a sus conocimientos en el arte de la medicina ${ }^{7}$.

La única fuente segura para delimitar la vida de este personaje y acercarnos, de forma más o menos certera, a su identificación son sus propias obras que, hasta la fecha, no han sido tenidas en cuenta por la comunidad científica. Y, sobre todo, la más importante de ellas, el Breviarium, que aún no ha conocido edición alguna. Johannes de Sancto Paulo fue autor de, al menos, cuatro tratados médicos: el citado Breviarium medicine; una obra De simplicium medicinarum virtutibus (también conocida como Virtus medicinarum y De conferentibus et nocentibus medicinis); unas Flores diaetarum $^{8}$ y un opúsculo sobre los días críticos. También se le han atribuido otras obras, entre ellas un comentario a las tablas farmacológicas del maestro Ricardo (Commentarium tabularum magistri Salerni ${ }^{10}$ ) y un De carnibus $^{11}$, pero la autoría de estos textos no ha sido verificada.

Por los datos obtenidos de sus obras podemos deducir que su formación médica fue adquirida en Salerno, donde escribió, si no todas, al menos parte de ellas, que pronto fueron conocidas hasta en la zona de influencia de Mont-

${ }^{7}$ El comentarista del De urinis, añade en este manuscrito de los primeros decenios del siglo XIII: Nam per artem artium eis dignitates et honores magnae praebendae adquirebantur, sicut Theophilo, Johanne de Sancto Paulo, Cardinali et episcopo Albano, et multis aliis legimus (K. Sudhoff, "Salerno, Montpellier und Paris um 1200", 53).

${ }^{8}$ Obra que se editó en 1574 en los Opera omnia de Bernardo de Gordon, a nombre de este autor (apud Guliel. Rovillium, ff. 948-63). Está inspirada en el Pantegni, Theorica, liber V y en el De particularibus diaetis de Isaac Iudaeus.

${ }^{9}$ A él alude Johannes de Sancto Paulo en el libro 5 del Breviarium, cf. El Escorial, Real Biblioteca del Monasterio, ms. lat. e.IV.22, f. 55v: et de temporibus egritudinis sufficienter scripsimus in tractatu meo de creticis diebus.

${ }^{10}$ Cf. L. Schuba, Die medizinischen Handschriften der Codices Palatini Latini in der Vatikanischen Bibliothek. Kataloge der Universitätsbibliothek Heidelberg, Band 1, Wiesbaden 1981, 543.

${ }^{11}$ Ms. Boston, Medical Library, 15, ff. 115r-20r. Cf. L. Thorndike-P. Kibre, A Catalogue of Incipits of Mediaeval Scientific Writings in Latin, Cambridge, Mass. 1963, 1543: Suo patri domino magistro Winoaldo... 
pellier y en París. Parece claro que trabajó en tiempos de Romualdo, cardenal y obispo de Salerno (muerto en 1181), dato que lo convierte en contemporáneo de Musandino y Ferrario y, probablemente, también de los tres autores de mayor calado teórico de Salerno: Mauro de Salerno, Gilles de Corbeil y Urso de Calabria. Por último, las referencias a los socii en su texto no dejan lugar a dudas sobre su dedicación profesional, de corte práctico más que teórico, en la línea de Plateario ${ }^{12}$.

De su obra más importante, el Breviarium, podemos extraer varios datos sobre su procedencia, modo de trabajar y las obras que sirvieron de fuente a este escritor médico. De su lectura podemos confirmar su adscripción salernitana. Por ejemplo, en el capítulo De variolis (lib. 1, cap. 12) el propio Sancto Paulo se identifica con los maestros de la escuela de Salerno:

Variole dicte sunt quod diversis maculis variant cutem ... cuius quatuor sunt species, una .... Tertia est in qua pustule crasse et nigre iiii vel $v$ in corpore appareat. hec ceteris periculosior, frequenter perveniens ex melancolia innaturali ex acutis febribus in quibus aduruntur humores; diciturque pustula, hanc nostri salernitani scabiam vocant.

En otro interesantísimo párrafo, también perteneciente al Breviarium, Sancto Paulo nos proporciona varios datos que nos permiten conocer mejor su actividad ${ }^{13}$; en primer lugar habla de sus fuentes y deducimos que es Constantino el Africano una de las principales. Pero el autor no se ha limitado a la transmisión del corpus constantiniano sino que, aun respetando la autoridad, es capaz de hacer una somera crítica sobre las características de la lengua del mítico y pionero traductor de Montecassino, lamentando la escasa competencia en lengua latina del africano. Al hilo de esta cuestión confiesa haber acudido a Galeno ${ }^{14}$ y nos informa sobre su método de trabajo, basado en la lectura constante y en la práctica aprendida de sus maestros, concretamente

${ }^{12}$ El hecho de que Plateario escribiera otro compendio médico, titulado Practica, o también Breviarium practice, es el responsable de que ambos autores se hayan confundido a lo largo de la tradición textual de ambas obras. Además, normalmente se conoce a Plateario con el nombre de 'Johannes', lo que también contribuyó a esta confusión entre ambos autores salernitanos. Un ejemplo de ello es el ms. München, Bayerische Staatsbibliothek, clm 75, s. XIV-XV, donde se ha conservado la obra de Plateario, que algunos estudiosos han confundido con la obra de Sancto Paulo (Cf. G. H. Krömer, Johanns von Sancto Paulo, 59).

${ }^{13}$ Sobre este fragmento ya llamó la atención V. Rose, Verzeichniss der Lateinischen Handschriften der Königlichen Bibliothek zu Berlin, Zweiter Band: Die Handschriften der Kurfürstlichen Bibliothek und der Kurfürstlichen Lande, Dritte Abteilung (Die HandschriftenVerzeichnisse der Königlichen Bibliothek zu Berlin, Dreizehnter Band), Berlin 1905, 1342-3.

${ }^{14}$ A quien cita varias veces, de manera especial en el explicit del Breviarium: Ecce iam breviter ut postulastis et in compendio signa causas et curas omnium egritudinum assig. navimus secundum disciplinas nostrorum auctorum et precipue Galieni. Vos secundum scripta ista operamini vestra opera dirigente deo omnipotente. 
del arzobispo Romualdo de Salerno. Sancto Paulo es consciente de que alguna puntualización a las fuentes, como en el caso de Constantino, podría acarrearle las críticas de sus lectores y se anticipa a ellas haciendo hincapié en su contraste con aquellas y su unión de teoría y práctica. De paso sabemos que componía a través de un copista personal, llamado Raynerius, que ponía por escrito el dictado del maestro:

(Lib. 5, cap. 6): Attendere oportet gradum... Ceterum dicit Constantinus in libro megategni in quo quedam verba Galeni exponit et se in huiusmodi resolvit. Quantitas inquid apponendorum litteris nullatenus denotari potest. Sufficit tamen ut apponatur secundum quod sibi propinquius est, et post pauca dicit. Assuefactio artis rerumque frequentatio que propinquiora sunt ostendunt, quarum quantitatis modum licet lingua explicare nequeamus, dicimus tamen ea que viciniora sunt. Et Constantinus cum esset africanus et in lingua latina pauper, explicare non poterat. Galienus autem in tegni evidentissime scripsit sic dicens. Si secundum calidius... Quantitatem complexionis et discrasie iam dixisse me verbis (o vobis) memini. Nam et Raynerius secundum quod a me audivit luculentissime scripsit. Sed quia liber iste ad plurimorum manus perveniet, nam necdum finitus summopere exoptatur, ideo reminisci hoc placet. Nec enodacionem meam reprehensione Constantini mei emuli criminentur, asserunt etenim et dicunt, Johannes de Sancto Paulo tota die legit et relegit, verum est equidem ut dicunt lego et relego lecta et relecta memorie commendo, verbis exprimo, litteris signo. Gratia dei in me operante per Jhesum Christum qui est benedictus in secula. Et que scribo non solum a libris verum etiam a peritis habeo magistris, precipue a Romoaldo venerabili per Dei gratiam Salernitanus sedis archiepiscopo.

En definitiva, sólo con el conocimiento de la tradición manuscrita del texto podremos llegar a conocer más a fondo la figura de este enigmático autor y su modo de trabajar, lo que nos vuelve a confirmar que el paso previo a cualquier otra consideración sobre los autores y sus obras requiere necesariamente una labor de crítica textual.

El BREVIARIUM DE SIGNIS, CAUSIS ET CURIS MORBORUM, UNA OBRA INÉDITA

El Breviarium de Johannes de Sancto Paulo es un compendio médico sobre las enfermedades y su curación, de corte eminentemente práctico, cuya edición crítica nos proponemos acometer. Cronológicamente fue compuesto cuando aún vivía el obispo Romualdo, mencionado en el texto arriba transcrito, es decir, antes de 1181. 
En cuanto a su estructura, la obra se divide en cinco libros, cuya materia se distribuye de la siguiente manera, como el mismo autor nos adelanta en el prólogo:
Assiduis petitionibus me karissimi compendiose morborum sig- na, causas et curas in scriptum redigere cogitis. Desideriis itaque vestris satisfaciens hunc laborem leto aggredior animo, consi- derans ipsum vobis simul et posteris fore quam maxime uti- lem. Exequar autem breviter signa et causas preponens et curas subiciens, ideoque breviarium intitulavi. Dividitur autem liber iste in $\mathrm{v}$ particulas, quarum prima de signis et causis et curis egritudinum universaliter tractatum continet et de exterioribus passionibus corporis. Secunda de passionibus animatorum mem- brorum et spiritualium. Tertia de passionibus nutritivarum par- tium. Quarta de morbis generativorum membrorum et scissure manuum et pedum. Quinta vero de febribus.

Efectivamente, el libro primero, con un total de veintiséis capítulos ${ }^{15}$, se dedica a los síntomas y causas de las enfermedades en su aspecto teórico y práctico, dando paso al tratamiento pormenorizado de todas las afecciones externas del cuerpo. A continuación, el libro segundo, en torno a veintinueve capítulos $^{16}$, trata de las enfermedades mentales y de la cabeza en general, las de la vista y el oído, la nariz, la boca y el aparato respiratorio. Los capítulos del libro tercero, unos veinticinco, se ocupan de las enfermedades del aparato gatrointestinal, las hepáticas y renales. En el libro cuarto, el más breve, con tan solo quince capítulos, se desglosan las enfermedades del aparato reproductor, tanto femenino como masculino, finalizando con las afecciones articulatorias. Por último, el quinto libro, de veintiséis capítulos, es una monografía sobre las fiebres.

El Breviarium se sitúa en el área de influencia del Viaticum de Ibn al Gazzar $^{17}$, una de las obras árabes traducidas por Constantino el Africano en el siglo XI, y por sus características es comparable a la Practica de Plateario, sin que hasta le fecha hayamos podido establecer los lazos de dependencia

${ }^{15}$ Para el desglose completo de los capítulos que componen cada uno de los libros, puede consultarse el realizado por V. Rose en su citada edición del Viaticum de Gilles de Corbeil (Leipzig 1907, 103-5), según el manuscrito de Erfurt Ampl. Q.207, y nuestro trabajo A. García González-A. I. Martín Ferreira, "El Breviarium de Johannes de Sancto Paulo y la materia médica en el manuscrito e.IV.22 de la Real Biblioteca de El Escorial", Actas del V Congreso Internacional de Latín Medieval Hispánico (Barcelona, 7-10 de septiembre de 2009), ed. J. Martínez Gázquez-O. de la Cruz Palma-C. Ferrero Hernández, en prensa.

${ }^{16}$ El número exacto depende de los manuscritos. No todos los códices presentan la misma división.

${ }^{17}$ Dice M. H. Green, "Johannes de Sancto Paulo", 286: "Indeed, the Breviary is the first Salernitan composition to attempt to match the Viaticum's nosological and therapeutic detail”. 
entre uno y otro compendio. Respecto a la fortuna posterior de esta obra, es palpable su influjo en autores como Gilberto Ánglico, en su Compendium medicinae $^{18}$. Sin embargo, en el contexto de la Escuela de Salerno, Sancto Paulo parece haber pasado más desapercibido, tal vez porque sus obras circularon a menudo a nombre de otros, como Constantino el Africano, Bernardo de Gordon ${ }^{19}$ o Plateario ${ }^{20}$.

\section{LOS MANUSCRITOS DEL BREVIARIUM}

Como ya hemos apuntado, estamos trabajando en la edición crítica de esta desconocida obra, y toda tarea de edición se inicia con la recopilación de sus testimonios manuscritos ${ }^{21}$. Hemos reunido un total de 35 testimonios manuscritos que hemos analizado de primera mano. El estudio directo de los manuscritos en unos casos ha refrendado los datos que de ellos ofrecían los catálogos especializados sobre obras técnicas y los catálogos de las diferentes bibliotecas, pero en otros muchos nos ha permitido constatar que estos catálogos presentaban datos erróneos en cuanto a la descripción, contenido, foliación o lecturas de los códices, errores que han sido convenientemente corregidos y actualizados en este trabajo. Así pues, estamos en condiciones de presentar el siguiente elenco de manuscritos que forman la tradición manuscrita del Breviarium de Johannes de Sancto Paulo:

1. Augsburg, Staats- und Stadtbibliothek, ms. $4^{\mathrm{O}}$ Cod. 26, ff. 188r-256r. Siglo XVex. Prologus ${ }^{22}$ : Assiduis peticionibus me karissimi compendiose morborum figuras et causas... Incipit: Quoniam longuitudini in hoc opere... Explicit: ...hoc scripta operavi vestra opera dirigente te omnipotente. Otros contenidos del códice: De medicinis simplicibus et compositis (ff. 1r-182v): [R]es particulares et sensibiles cum quibus medici operantur possunt cognosci tribus viis: fraccione experimento et meditacione antiquorum... (f. 11r) Asaru grece aerma ara baccara latine... - ...grano quod reperitur in Cassias et Almans sive Rasis ponit istas siliquas in tractatu de trocistis; Índice del libro V del Canon de Avicena (ff. 183r-4v); Voca-

${ }^{18} \mathrm{Cf}$. Compendium medicine Gilberti anglici tam morborum universalium quam particularium nondum medicis sed et cyrurgicis utilissimum, Lugduni 1510.

${ }^{19} \mathrm{El}$ tratado titulado Flores diaetarum aparece atribuido a Bernardo de Gordon en sus Opera omnia, Lugduni 1574, 948-63. A su vez, el Liber de simplicium medicinarum virtutibus se publicó a nombre de Constantino el Africano en Omnia Opera Ysaac, Lugduni 1511.

${ }^{20}$ Como podrá verse en el elenco de manuscritos, en algunos de ellos la obra se atribuye a Plateario, por ejemplo en el citado ms. e.IV.22 de la Real Biblioteca de El Escorial.

${ }^{21}$ En las tareas de localización de los códices hemos recibido la generosa ayuda de la profesora Monica H. Green, de la Arizona State University (USA). Nos ha permitido obtener datos de sus trabajos mientras todavía estaban inéditos. Uno de ellos ha sido ya publicado: M. H. Green, "Rethinking the Manuscript Basis of Salvatore de Renzi's Collectio Salernitana: The corpus of medical Writings in the "long” Twelfth Century", La Collectio Salernitana di Salvatore De Renzi, a cura di D. Jacquart e A. Paravicini Bagliani, Firenze 2008, 15-60.

${ }^{22}$ En todos los textos que se reproducen en el presente trabajo nos limitamos a trasladar fielmente la lectura del códice. 
bularius herbarum secundum ordinem alphabeti (latín-alemán, ff. 185r-7v): Arthemisia - byfuss... - ... zuccorum - zucker; Simplices et compositae medicinae in apothecis per ordimen alphabeti (ff. 257r-268v): Antimonium ... Composita: Sirupus rosarum... (f. 265v) ${ }^{23}$.

2. Basel, Öffentliche Universitätsbibliothek, ms. D.II.33, ff. 1r-32r. Siglo XIII. Prologus: Assiduis peticionibus me carissimi compendiose morborum signa, causas et curas... Incipit: Incipit breviarium. Quoniam longuitudini in hoc opere parcere destinavimus... Explicit: ...vos secundum scripta ista operamini vestra opera dirigente Deo omnipotente. Explicit breviarius. Otros contenidos del códice: Varias recetas (f. 32v: Aqua vitae super omnia vim habens sic debet fieri...; f. 33r: De dolore capitis: aloe, agaricus, absinthium... De melancolia... De frenesi... De ethica...); Platerius Matthaeus, De simplicitus sc. Circa instans: Negocium [de] simplicibus medicinis nostrum versatur propositum simplex autem medicina est... (ff. 36v-71v); Copho, Anathomia porci: Incipit anathomia... (ff. 71v3v); Johannes Platearius, Practica brevis: Incipit practica magistri Platerii... - ...pulvis inde factus cum licinio fistule immittatur (ff.73v-102v); Alchandrus [sic], De nominibus: Accipiat nomen suum et maternum... ...apostolorum (ff. 103r-8v) ${ }^{24}$.

3. Basel, Öffentliche Universitätsbibliothek, ms. D.III.3, ff. 86ra-120rb. Siglo XIII. Prologus: Assiduis peticionibus karissimi me compendiose morborum signa, causas et curas in scriptum redigere cogitis... Incipit: Quoniam longuitudini in hoc opere parcere destinavimus... Explicit: ... vos secundum scripta ista operamini vestra opera dirigente Deo omnipotente. Explicit liber breviarii. Qui scripsit scribat semper cum domino vivat. Otros contenidos del códice: Constantinus Africanus, Viaticum: Quoniam quidem ut in rethoricis... (ff. 1r-35r); Const. Afr., Liber Pantegni, pars II Practica: Quia in prima parte nostri Pantegni...(ff. 35r-49r); Const. Afr., Liber de gradibus: De gradibus primo quid sint... (ff. 49r-53v); Commentarius in Isagogas Johannitii ad librum Tegni Galeni a m[agistro] B[artholomaeo]: Duplex ordo doctrine rebus tractandis... (ff. 54r-74r); Liber isagogarum ad Tegni Galeni: Desiderium sciendi mentibus humanis... (fragm. ff. 74r-v); Const. Afr., Liber de gradibus (comment.): Liber iste graduum tercia pars... (fragm. ff. 75r-6r); Bartholomaeus Salernitanus, Commentarius in prognostica Hippocratis: Tria sunt medicine... (ff. 77r-85v); Tabula medicinarum, quid pro quo: In primo pabulo salis medicinalis... (ff. $120 \mathrm{v}-1 \mathrm{v})^{25}$.

${ }^{23}$ W. Gehrt, Die Handschriften der Staats- und Stadtbibliothek Augsburg $4^{\circ} \mathrm{Cod}$ 1-150. Handschriftenkataloge der Staats- und Stadtbibliothek Augsburg; Bd. 6. Wiesbaden 1999, 44-5.

${ }^{24}$ M. Steinmann, Die Handschriften der Universitätsbibliothek Basel: Register zu den Abteilungen C I - C VI, D - F sowie zu weiteren mittelalterlichen Handschriften und Fragmenten, Basel 1998, 416.

${ }_{25}$ M. Steinmann, Die Handschriften der Universitätsbibliothek Basel, 416; V. Rose, 
4. Berlin, Staatsbibliothek zu Berlin-Preussischer Kulturbesitz, ms. lat. qu. 533 (Phillipps 20601), ff. 1-85. Siglo XIV. Prologus: Non legitur. Sequuntur tituli capitulorum totius operis: Incipiunt capitula prime particule. De generalibus signis. De causis egritudinum... Incipit: (f. 2) [Quoniam lo] nguitudini in $h[o c] .$. Explicit: ... Sit autem dieta tenuis quia cito venit egritudo ad statum, et attenuantur vires egri sicut prius diximus [En este manuscrito el Breviarium concluye con estas palabras del capítulo $D e$ causonide del libro 5. El explicit acostumbrado (...vos autem secundum scripta ista operamini nostra opera dirigente Deo omnipotente. Amen) se encuentra en el f. 85, ya que se ha producido una variación en el orden de los capítulos finales de la obra]. Otros contenidos del códice: tras el Breviarium se han añadido dos pequeños escritos titulados De signis acutarum febrium (f. 88-91) y De creticis diebus (f. 91-5) ${ }^{26}$.

5. Bethesda, Maryland, National Library of Medicine, ms. 511.1, ff. 1r121v. Siglo XIII: Italia (?), scr. Arnaudus. Prologus: Assiduis peticionibus me karissimi compendiose morborum signa, causas et curas in scriptum redigere cogitis... Incipit: Quoniam longuitudini in hoc opere parcere destinavimus... Explicit: ...vos autem secundum scripta ista operamini opera vostra dirigente deo patre omnipotente qui vivit et regnat per omnia secula seculorum. Amen. Qui lybrum ystum scripsit, plures scribere possit, scilicet arnaudus. Otros contenidos del códice: el manuscrito está enteramente dedicado a la transmisión del Breviarium de Johannes de Sancto Paulo, aunque éste comienza en el f. $3 r$. En el f $1 \mathrm{r}-\mathrm{v}$, tal vez unido a modo de tapas, sólo se distingue un texto a dos columnas y mutilado que concluye con el nombre de Joannes de Gadesden angli[icus] añadido por una mano posterior ${ }^{27}$.

6. Bruxelles, Bibliothèque royale de Belgique (KBR), ms. 11456-11464, ff. 139r-201v. Siglo XIV in.: Heverlee, Park. Prologus: Incipit prologus super breviarium compositum a Iohanne de sancto Paulo. Assiduis peticionibus me karissimi compendiose morborum signa, causas et curas in scriptis redigere cogitis. Incipit: Quoniam longitudini in hoc opere parcere earum egritudinum destinavimus...Explicit: Vos secundum scripta ista operamini vestra opera dirigente deo omnipotente. Explicit liber. Otros contenidos del códice: Johannitius-Hunain ibn Ishaq, Isagoge: Medicina dividit... (ff. 1r-13v); Hippocrates, Aphorismi:Vita brevis, ars longa... (ff. 15r-29r), Prognostica: Omnis qui mediatu... (ff. 29r-37v), De regimine acutorum: Quidam egrotantium... (ff. 38r-48v); Galenus, Ars parva: Tres

Egidii Corboliensis Viaticus, 103; L. Thorndike-P. Kibre, A Catalogue of Incipits, 153.

${ }^{26}$ V. Rose, Verzeichniss der Lateinischen Handschriften der Königlichen Bibliothek zu Berlin, 1342-3.

${ }^{27}$ E. Tunis, Early Western Manuscripts in The National Library of Medicine. A ShortTitle List, Bethesda, Maryland 1989, 4; L. Thorndike-P. Kibre, A Catalogue of Inci-pits, 153. 
sunt omnes doctrine... (ff. 50r-84v); Theophilus Protospatharios, De urinis: De urinarum differentia... (ff. 85r-97r), De pulsibus (ff. 97r-100r); Isaac Judaeus-Ishaq al-Israili, Liber de gradibus simplicium: Quoniam disputationem... (ff. 101r-24v); Johannes de Sancto Paulo, Liber de simplicium medicinarum virtutibus: Cogitanti michi de simplicium... (ff. 125r-38v) ${ }^{28}$.

7. Cambridge, University Library, ms. 938 (Ee.II.20), ff. 219ra-40va. Siglo XIV. Prologus: Assiduis peticionibus me karissimi compendiose morborum signa, causas et curas... Incipit: Quoniam longuitudini in hoc opere parcere destinavimus... Explicit: ...opera vestra dirigente Deo omnipotente. Explicit Liber Breviarii secundum Magistrum Johannem de Sancto Paulo. Otros contenidos del códice: Glossa super Viaticum Constantini: Cum omnia ex quatuor elementa generata... (ff. 1-12); Tractatus de modo medendi: In medicinis corporibus et maxime purgandis... (ff. 137b); Pomum Ambre: [P]omum Ambre duplicant ad renum... (ff. 17b-24a); Repressiva Magistri Ricardi: Aloe quia a prima litera incipit erit principium in repressiva... (ff. 24a-7b); Prescripciones: De capillis, et primo de tinea capillorum... (ff. 27b-40a); Summa Ricardi de signis morborum: Filius meus, duntaxat in laudabilibus, existet... (ff. 40b-54a); Tractatus de pulsibus: Cogitanti mihi nostram vocationem... (ff. 54b-60); Liber de simplici medicina: Cogitanti mihi de simplicium medicinarum virtutibus... (ff. 61-5a); Liber de gradibus simplicium medicinarum: Quoniam simplicis medicie disputationem... (ff. 66-75); Aegidius de urinis: Dicitur urina quoniam fit a renibus $u[r]$ na... (ff. 78-101); Liber de urinarum differentia (ff. 102-24a); Tractatus magistri Ysaac de febribus: Quoniam te sivi Carissime Johannes...(ff. 128-69): Liber magistri Ferrarii de febribus (ff. 171-80); Practicae Archimachei et Rogeri (ff. 188-218a); Tractatus de flebotomia (ff. 218a-21); Practica summa Gerardi: Cum omnis scientie ex fine et utilitate (ff. 222-8); Breviarium Platearii: Assiduis peticionibus me carissimi... (ff. 230-51) ${ }^{29}$.

8. Cambridge, University Library, ms. 1133 (Ee.VI.41), ff. 4r-122v. Siglo XIV. Prologus: Incipit Breviarium magistri Johannis de Sancto Paulo.

${ }^{28}$ Catalogue des manuscrits de la Bibliothèque Royale des Ducs de Bourgogne, tom. I, Bruxelles-Leipzig 1842, 230; R. Calcoen, Inventaire des manuscrits scientifiques de la

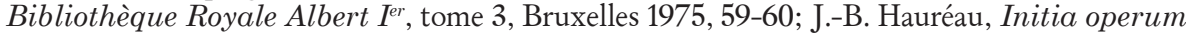
scriptorum Latinorum Medii Potissimum Aevi ex codicibus manuscriptis et libris impressis, 6 vols., 2 append., Turnhout 1973, app. 1, f. 53 von Sancto Paulo, 59.

${ }^{29}$ A Catalogue of the Manuscripts preserved in the Library of the University of Cambridge. Edited for the Syndics of the University Press, vol. 2, Cambridge 1857 (reimpr. Hildesheim 1980), 35-8 (La descripción aquí presentada está tomada de este catálogo en el que, sin embargo, las referencias de los folios que contienen cada una de las obras no se corresponde con la paginación real del manuscrito, como puede verse en el caso del Breviarium de Johannes de Sancto Paulo, al que el catálogo titula Breviarium Platearii); V. Rose, Egidii Corboliensis Viaticus, 103; L. Thorndike-P. Kibre, A Catalogue of Incipits, 153, 1284; G. H. Krömer, Johanns von Sancto Paulo, 59. 
Assiduis peticionibus me karissimi socii compendiose morborum signa et causas et curas... Incipit: (f. 4v) Quoniam longuitudini parcere destinavimus in hoc opere... Explicit: ...et vestram operam dirigite Deo omnipotente. Explicit. Otros contenidos del códice: Tractatus de simplici medicina sc. Circa instans: Circa instud negocium in simplicibus medicinis nostrum versatur propositum... (ff. $122 \mathrm{v}-237 \mathrm{r})^{30}$.

9. Cambridge, University Library, ms. Additional 6865, ff. 14r-60rb. Siglo XII-XIII, c. 1200. Prologus: Incipit breviarius a magistro Johanne de Sancto Paulo editus. Assiduis peticionibus me karissimi compendiose morborum signa, causas et curas... Incipit: Quoniam longuitudini in hoc opere parcere destinavimus... Explicit: ...vestra opera dirigente Deo omnipotente. Explicit liber $4^{u s}$ feliciter. Otros contenidos del códice: Homiliae $1^{\mathrm{a}}-14^{\mathrm{a}}$ (ff. 1r-11v); De 4 elementis et 4 animalibus: Nota quod sunt quatuor animalia que pascuntur ex quatuor elementis... (f. $12 \mathrm{v}-3 \mathrm{r}$ ); notae (f. 13v); Antidotarium Nicolai: Aurea Alexandrina dicta est ab auro... (ff. 60v79vb); De ponderibus et mensuris (f. 79v); Synonima Antidotarii Nicolai: Artemisia id est matricaria... (ff. 79vb-81rb); Nicolai de Hostresham Practica: Caput multis passionibus diversisque humoribus uel in existentibus... (ff. 81v-103v); De matrice: Matrix ad humanam generationem membrum maxime multis subiacens passionibus... (ff. 103va-6vb); De sciatica: Passio sciatica plurimum fit ex humoribus... (f. 106vb); Cum sit stomachus pro nature necessitate / Febris autem diffinitionem brevitatis causa / Cum stomachus pro... (ff. 107vb-8rb); De febribus: Febris autem diffinitionem brevitatis causa... (ff. 108r-15vb); De pleumonia: Pleumonia est apostema nascens circa pulmonem... (ff. 115vb-116ra); De syrupis alterativis constrictoriis et laxativis: Cum multifaria habeatur siruporum divisio... (ff.116ra20r); De oleis: Postquam dictum est de syrupis consequenter adiungimus de oleis... (ff. 120r-3v); De aquis: Dictum est sufficienter de oleis sed quoniam aquarum nonnullis... (ff. 123v-30r); De clisteribus suppositoriis syringis et pessariis: Clisterium quatuor sunt genera mollificativum / De suppositoriis de syringis de pessariis... (ff. 130r-3r)

10. Darmstadt, Hessische Landes- und Hochschulbibliothek, ms. 329, ff. 148va-92va. Siglo XIII-XIV. Monasterio de Sankt Jakob-Lüttich: Liber Monasterii sancti Jacobi leodiensis, según una mano del s. XIV. Prologus: Assiduis peticionibus me karissimi compendiose morborum signa... Incipit: Qvoniam longitudini in hoc opere... Explicit: ...operamini nostra opera dirigente Deo omnipotente. Otros contenidos del códice ${ }^{32}$ : Ga-

${ }^{30}$ A Catalogue of the Manuscripts preserved in the Library of the University of Cambridge, 278; L. Thorndike-P. Kibre, A Catalogue of Incipits, 1284. G. H. Krömer, Johanns von Sancto Paulo, 59.

${ }^{31}$ Descripción reconstruida por M. H. Green a partir de los datos ofrecidos en el catálogo de L. Thorndike-P. Kibre, A Catalogue of Incipits... ya citado en numerosas ocasiones.

${ }^{32}$ Se trata de un códice facticio compuesto de tres manuscritos. 
riopontus, Passionarius (ff. 1r-90v); De chirurgia (ff. 91ra-100rb); Copho, De modo medendi: De modo medendi quot et quibus modis ... ego hoc opus de modo medendi a cofonis ore et iohanne de platea suorumque sociorum compendiose collegi ... seminis eruce. maiorane denter 3 III (ff. 104ra-15ra); Johannes Platearius, Practica: Amicum induit. Qui iustis amicorum precibus ... talis pulvis cum licinio inmittatur. Explicit liber platearii (ff. 115ra-48rb); Glossae (ff. 193r-4r) ${ }^{33}$.

11. El Escorial, Real Biblioteca del Monasterio de San Lorenzo de El Escorial, ms. lat. e.IV.22, ff. 1r-56v. Siglo XIII in.: Francia (?), propiedad del médico Jean Jacques Chifflet (1588-1660). Prologus: Incipit prologus breviarii Ioannes de Sancto Paulo. Assiduis animatum petitionibus me karissimi compendiose morborum signa, causas et curas in scriptum redigere cogitis... Incipit: Quoniam longitudini in hoc opere parcere destinavimus... Explicit: ... factum de psillio et aqua psillii ponatur (En este manuscrito de El Escorial el Breviarium se encuentra incompleto faltándole los trece últimos capítulos del libro V). Otros contenidos del códice: el resto de este códice facticio (ff. 57r-137v) se dedica a la recopilación de 989 recetas y consejos médicos cuyo compilador agrupó según la tipología de los fármacos o de las patologías para las que estaba indicada cada una de las fórmulas. Entre estos escritos se encuentra también, en los ff. 67r-73r, el tratado De urinis de Ricardo Ánglico ${ }^{34}$.

12. Erfurt, Universitäts- und Forschungsbibliothek Erfurt/Gotha, ms. Ampl. Q.207, ff. 118r-79r. Siglo XIV in.: procedencia meridional. Prologus: Assiduis peticionibus me, karissimi, compendiose morborum signa... Incipit: Quoniam longitudini in hoc opere... Explicit: ...operamini nostra opera dirigente Deo omnipotente. Otros contenidos del códice: Liber Platearii de simplicibus medicinis: Circa instans negocium in simplicibus medicinis nostrum versatur propositum ... hoc lecto fine concludamus (ff. 2r-50r); Liber Isaac de urinis: In Latinis quidem libris nullum auctorem invenire ... uvea dolorem renum significat (ff. 50v-75r); Mauri magristri tractatus de urinis: Quoniam de urinarum sciencia dicturi sumus, primo vedendum ... si mediam medietas, si vero totum, non minuetur (ff. 75v-91r); Excerptum de libro $2^{\circ}$ canonis Avicenne de simplicibus medicinis: Quoniam, ut ait philosophus omnia memorie commendare ... tyriaca morsibus venenosis et similiter tauri $(\mathrm{ff} .92 \mathrm{r}-117 \mathrm{v})^{35}$.

${ }^{33}$ F. Edward Cranz, A Microfilm Corpus of Unpublished Inventories of Latin Manuscripts through 1600 A.D., New London, Conn. 1987, 26; V. Rose, Egidii Corboliensis Viaticus, 103 .

${ }^{34} \mathrm{G}$. Antolín, Catálogo de los códices latinos de la Real Biblioteca de El Escorial, vol. II, Madrid 1911, 122-3; A. García González-A. I. Martín Ferreira, "El Breviarium de Johannes de Sancto Paulo", en prensa.

${ }^{35}$ W. Schum, Beschreibendes Verzeichniss der Amplonianischen Handschriften-Sammlung zu Erfurt, Berlin 1887, 465-6; G. H. Krömer, Johanns von Sancto Paulo, 59; L. 
13. Exeter, Dean and Chapter Library, ms. 3526, ff. 1r-47r. Siglo XIII in.: Inglaterra (?). Prologus: Assiduis peticionibus me karissimi compendiose morborum signa... Incipit: Quoniam longitudini in hoc opere parcere destinavimus... Explicit: ...vestra opera dirigente Deo omnipotente. Otros contenidos del códice: el último folio del códice (f. $47 \mathrm{rv}$ ) recoge varias recetas ginecológicas en 24 parágrafos, una de ellas titulada ad concipiendum masculum $^{36}$.

14. Firenze, Biblioteca Medicea Laurenziana, ms. Plut. 73, cod. 33, ff. 17r50r. Siglo XIII: Italia, en el margen f. 1v se lee Magistri Antonii Landucii. Prologus: Incipit breviarium magisti Joannis de Sancto Paulo. Assiduis peticionibus me karissimi compendiose morborum signa, causas et curas in scriptum redigere cogitis... Incipit: Quoniam longitudini in hoc opere parcere destinavimus... Explicit: ...Vos secundum scripta ista operamini vestra opera dirigente Deo omnipotente. Explicit breviarium magristi Joannis de S. Paulo. Otros contenidos del códice: Tractatus de urinis: Quoniam de urinarum scientia tractaturi sumus... (ff. 1r-16r); Quaedam Chirurgica: Quoniam scarificatio alia fit cum incisione... (ff. 50v-1r); De signis mortalibus in aegroto et de diebus criticis: Omnis significatio in infirmo... (ff. 51r-8r); Contra malum mortuum purgatio: Exsistente bene corpore, bis, aut ter... (ff. 58r-9r); Liber de febribus: De signis, causis et curis egritudinum particulariter tractaturi... (ff. 59r-77r); De phlebotomia Mauri: Presentis negocii propositum est breviter... (ff. 77r-80v); Tractatus de anatomia: Galieno attestante in Tegni... (ff. 80v-85r); Hippocras de regimine medicorum: Consideranti mihi votum bonum, votum castum... (ff. 85r-91r); Praescriptiones et remedia: Vnguentum Aragomu, idest aiutorium... (ff. 91r-2r); De cibis et potibus: De cibis et potibus preparandis infirmis...(ff. 92r-5r); Rolandi Parmensis Chirurgia: Medicina equivocatur ad duo ... Chirurgia dicitur a ciros... (ff. 95r-136v) ${ }^{37}$.

15. Hildesheim, Dombibliothek, ms. 749, ff. 119r-64v. Siglo XIII ex. Prologus: Assiduis peticionibus me karissimi ... Exequitur autem breviter signa et causas preponens et curas subiciens. Ideoque breviarium intytulavi... Incipit: Quoniam longitudini in hoc opere parcere destinavimus... Explicit: ...per omnia secula seculorum amen. Explicit breviarium breviter compilatum. Otros contenidos del códice: Bartholomaei Salernitani

Thorndike-P. Kibre, A Catalogue of Incipits, 1284.

${ }^{36}$ N. R. Ker, Medieval Manuscripts in British Libraries, vol II, Oxford 1977, 836; M. P. Crighton, A Catalogue of the Medical Books and Manuscripts including a selection of the scientific works in Exeter Cathedral Library, Birmingham 1934, 193; P. W. Thomas, Medicine and science at Exeter Cathedral Library: a short-title catalogue of the printed books, 1483-1900, with a list of 10th- to 19th century manuscripts, Exeter 2003, 282.

${ }^{37}$ A. M. Bandini, Catalogus codicum Latinorum Bibliothecae Mediceae Laurentianae, tomus III, Florentiae 1776, 60-4; G. H. Krömer, Johanns von Sancto Paulo, 59; L. Thorndike-P. Kibre, A Catalogue of Incipits, 1284. 
Practica: Practica dividitur in duo... (ff. 4r-67r); Medicina ad aves custodiendas (ff. 67v-8r); De paralysi (ff. 68r-v); Johannis Afflacii liber aureus: $S i$ dolor capitis sit ex causa... (ff. 69r-98v); Johannis de Parma practicella ex dictis Mesue abbreviata: Quoniam quidam de melioribus amicis... (ff. 99r100v); De generibus pulsuum / De coloribus urinarum (ff. 100v-2r); Aegidii Corboliensis de urinis: Dicitur urina quoniam... (ff. 102r-4r); Archimathei Modus medendi (ff. 104v-9v); Versus de diebus criticis / Versus medicinales ex regimine sanitatis Salernitano: Quatuor humores humano corpore... (f. 109v); Roger de Baron, Practica parva: Humana natura non minus... (ff. $110 \mathrm{r}-4 \mathrm{v})$; varios pequeños tratados que el catálogo titula: De diebus criticis, [Johannes Stephanus] De dosibus medicinarum, De coitu, Regula ad sanguinem minuendum, Definitiones physico-medicae, Regulae sanitatis, Regimen sanitatis per duodecim menses, De urina, Arnaldus de Villanova (?): De phlebotomia, recetas (ff. $114 \mathrm{v}-8 \mathrm{v})^{38}$.

16. Leipzig, Universitätsbibliothek, ms. 1140, ff. 10rb-57vb. Siglo XIVex.: Alemania (?). Prologus: Incipit breviarium magristi Joannis de Sancto Paulo. Assiduis petitionibus me karissimi socii compendiose morborum signa et causas et curas in scriptis redigere cogitis... Incipit: Quoniam longitudini in hoc opere parcere destinamus... Explicit: ...Item aliis autem $x x$ horis ex altera tamen (La obra se interrumpe en el capítulo De emitriteo minori del libro $\mathrm{V}$ faltándole los cinco últimos capítulos). Otros contenidos del códice: Nomima aegritudinum: Tractatus de functionibus infirmitatum... (ff. 1r-2r); Avicennae de urinis: Excerptum de vrinis ex Avicenna... (ff. 2r-7r); sentencias y recetas (ff. 7r-10r); Gerardus de Montepessulano (Cremonensis ?), Summa de modo medendi: Incipit tractatus signorum quatuor humorum... (ff. 58r-87v); De medicinis laxativis compositis: Expleto tractatu medicinarum... (ff. 82v-5v); De apporimatis medicinarum (ff. 85v-7r); dos tratados de urinis (ff. 87v-94r); Gualterius Agulinus, Liber pulsuum: Cum decem sunt genera pulsuum... (ff. 94r-95v); Nomina antidotorum et egritudinum (ff. 95v-101r); Tractatus de collectione medicinarum (ff. 101r-2v); fragmento del Liber primus Canonis Avicennae (f. 102v); Franciscus Lambardes (?), Tabula de urinis: Colores significantes indigestionem... (ff. 103v-7r).

17. Leipzig, Universitätsbibliothek, ms. 1171, ff. 1ra-23rb. Siglo XV: monasterio cisterciense de Altzelle (Nossen-Dresde), al pie de los ff. $1 \mathrm{v}-2 \mathrm{r}$ puede leerse Liber monasterij Veteriscelle, repositus ad Bibliothecam pubblicam Anno 1506. Prologus: Assiduis peticionibus me karissimi compendiose signa causas curas in scriptum redigere cogitis... Incipit: Quoniam longitudini in hoc opere parcere volumus... Otros contenidos del códice: según se lee en el reverso de las tapas del códice, éste contiene además, tras el

${ }^{38}$ R. Giermann-H. Härtel-M. Arnold, Handschriften der Dombibliothek zu Hildes-heim, Teil 2: Hs 700-1050, St. God. Nr. 1-51, Ps 1-6, J 23-95, Wiesbaden 1993, 82-5. 
Breviarium de Johannes de Sancto Paulo, un Tractatus de clisteris, sirupis et aquis artificialibus et oleis, una Practica Johannicii, otra Practica Bartholomei y unos Dicta super Egidium de urinis ${ }^{39}$.

18. London, British Library, ms. Additional 16385, ff. 4v-78v. Siglo XIII. Prologus: Incipit breviarius a magistro Iohanne de sancto paulo editus. Assiduis peticionibus me karissimi socii compendiose morborum signa, causas et curas in scriptum redigere cotigis. Incipit: De generalibus signis et causis egritudinum. Quoniam longitudini in hoc opere parcere destinavimus... Explicit: ...vos autem secundum scripta ista operamini. Opera vestrum dirigente domino deo omnipotente qui vivit et regnat per omnia secula secoluroum. Amen. Explicit. Otros contenidos del códice: Tractatus de confectione medicinarum (ff. 1r-4v); Tractatus de clisteribus (f. 79r); Libellus de modis medendi (ff. 79r-91r); Curae magistri Matthaei Ferrarii, de febribus (ff. 91v-121r); Regulae urinariae magistri Matthaei Ferrarii $(\text { ff. } 121 \mathrm{v})^{40}$.

19. London, British Library, ms. Sloane 418, ff. 327r-8r. Siglo XV. Prologus: No tiene. Incipit: Apostema in stomacho et intestinis interdum fit ex calidis humoribus et interdum ex frigidis... Explicit: ...Si ex calore de succo solatri, aqua rosata, oleo rose et viole, sandali, aliter ex ruta et camphora potus sit aqua rosata vel pluviali (Se trata de un fragmento de la obra, ya que sólo contiene dos únicos capítulos del libro III del Breviarium; en concreto, los capítulos De apostemate stomachi y De diarria). Otros contenidos del códice: en este códice el fragmento de Johannes de Sancto Paulo se ha transmitido, entre otras obras, con la Parva summa del maestro Rogerio (expl. f. 325r), un Tractatus de clisteribus (ff. 325v-6v) y las Tabulae Salerni (ff. 328v y ss.) ${ }^{41}$.

20. London, British Library, ms. Sloane 2454, ff. 53v-81v. Siglo XIII ${ }^{\text {ex. }}$. Prologus: Assiduis petitionibus me Karissimi compendiose morborum signa, causas et curas in scriptum redigere cogitis... Incipit: Quoniam longitudini in hoc opere parcere destinavimus, ne pro nimia brevitate generemus obscuritatem... Explicit: ...Vos igitur secundum ista scripta operamini, domino opera vestra dirigente Amen. Explicit hic breviarius $^{42}$. Otros contenidos del códice: Johannis Platearii Salernitani, Practica

${ }^{39}$ Agradecemos al Dr. Almuth Märker y a la Dra. Anette Löffter, de la Universitätsbibliothek Leipzig, que nos hayan proporcionado tan amablemente las descripciones de estos dos manuscritos de Leipzig.

${ }^{40}$ Catalogue of Additions to the Manuscripts in the British Museum in the years 1846-1847, London 1864, 186; V. Rose, Egidii Corboliensis Viaticus, 103; L. Thorndike-P. Kibre, A Catalogue of Incipits, 153.

${ }^{41}$ E. J. L. Scott, Index to the Sloane Manuscripts in the British Museum, London 1904, 425: bajo en nombre de Platearius (Joannes) al. Sco. Paulo (Joannes de); L. Thorndike-P. Kibre, A Catalogue of Incipits, 114.

${ }^{42}$ La obra se encuentra atribuida en este manuscrito a Johannes Dama[scenus] (?): Incipit breviarius magistri Johannis Dama. 
brevis morborum curandorum, etiam febrium (ff. 2r-25v); Practica magistri Bartholomei luculentissimi philosophi: Medicina dividitur in duo; scilicet, in scientiam conservativam sanitatis et curativam aegritudinis... (ff. 26r-52r); Liber Constantini de differentia inter animam et spiritum, translatum ab Johanne Hispaniensi: Incipit prologus: Interrogasti me, (honoret te Deus!) de differentia inter spiritum et animam, et ut tibi scriberem quod dixerunt antiqui in ea; et ecco scribo tibi quaedam collectiva quae excerpsi de libro Platonis... Incipit tractatus: Spiritus est quoddam corpus subtilo quod in humano corpore oritur... (ff. 82r-84r); Tractatus de membris corporis humani: Cerebrum natura frigidum et humidum est; ideo ut facilius ad susceptionem omnium humorum contrahatur... (ff. $84 \mathrm{v}-86 \mathrm{r})^{43}$.

21. London, Royal College of Physicians, ms. 223a, ff. 26v-50r. Siglo XIII ${ }^{\text {in: }}$ Francia. Prologus: [Ass]iduis peticionibus me karissimi compendiose... Explicit: ...operamini opera vestra domino nostro adiuvante. Amen. Otros contenidos del códice: Platearii de simplicibus medicinis sive Circa instans: Circa instans negocium in simplicibus medicinis ... nisi propter diuturnam decoctionem (ff. 1r-26r); Johannes Platearius, Practica brevis: Amicum induit qui iustis ... non vehementi rubori modico (incompleto: ff. 50r-68v); parte de un documento del siglo XIV con nombres en caso dativo: ...priori prioratus de monasterio lons ... marie de chanloto ... Regnando de ponteheberti... (f. $69 \mathrm{v})^{44}$.

22. Milano, Biblioteca Ambrosiana, ms. G 30 sup., ff. 1r-68r. Siglo XIII. Prologus: Assiduis petitionibus me karissimi... Incipit: Quoniam longitudini in hoc opere parcere destinamus... Explicit: ...vestra opera dirigente Deo omnipotente. Otros contenidos del códice: Curas Petroncelli (f. $68 \mathrm{r}-106 \mathrm{v})^{45}$.

23. Montpellier, Biblioteca de la Faculté de médecine, ms. 318, ff. 39r-75v. Siglo XII-XIII. Prologus: Assiduis petitionibus me karissimi morborum signa ... [D]e fissura manuum et pedum. Incipit: Quoniam longitudini in hoc opere parcere destinamus... Explicit: ...ista operamini vestra opera dirigente Deo omnipotente. Amen. Otros contenidos del códice: Tractatus medicinae: Quoniam inter omnia curationum genera medendi modus... (ff. 1r-5r), De urinis. Ordo rerum exigit sequentium ut de urinis... (ff. $5 \mathrm{r}-$ 6v), Guillelmus Pictaviensis, Liber urinarum: Urina ergo tenuis et rubea ...

${ }^{43}$ E. J. L. Scott, Index to the Sloane Manuscripts, 41, 134, 281, 424; L. Thorndike-P. Kibre, A Catalogue of Incipits, 153.

${ }^{44}$ N. R. Ker, Medieval Manuscripts in British Libraries, vol. 1, Oxford 1969, 198-199.

45 Inventario Ceruti dei manoscritti della Biblioteca Ambrosiana, Milano 1977, 540; F. Bazzi, "Catalogo dei manoscritti e degli incunaboli di interesse medico-naturalistico dell'Ambrosiana e della Braidense", Quaterni di "Castalia" 7, 1961, n 374 y 607; L. Thorndike-P. Kibre, A Catalogue of Incipits, 153,6 y 1284,6; Petrocellus, Practica, traduzione e commento a cura di A. Capparoni, Roma 1958. 
et ypostasim albam inferius (ff. 6v-8r), Quatuor sunt elementa... (ff. 8r9v), Ad pectoris structuram... Si dolor fuerit in stomacho... Experimentum expertum ad vicium pulmonis... Pullule contra raucedinem... Ad menstrua provocada... (f.9v); Constantinus Africanus, Liber maior de coitu: [C]reator volens animalium genus firmiter permanere et non perire... (ff. 10r-3v); Bartholomeus Salernitanus, Practica: [P]ractica dividitur in duo in scientiam conservativam sanitatis... (ff.14r-38v ${ }^{46}$.

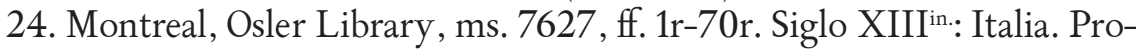
logus: Incipit breviarium Ypochratis. Assiduis peticionibus me karissimi compendiose signa morborum causas et curas in scriptum redigere cogitis... Incipit: Quoniam longitudini in hoc opere parcere destinavimus... Explicit: ...Vos secundum scripta ista operamini opera nostra dirigite Deo omnipotente. Amen. Otros contenidos del códice ${ }^{47}$ : Recetas y notas médicas (ff. 70r-8v): Vnguentum ad scrophulas... (f. 70r), Theorica est perfecta noticia rerum... (f. 71v), Vnguentum ad lentigines... (f. 77r), De cura artetice... (f. $77 \mathrm{v})^{48}$.

25. München, Bayerische Staatsbibliothek, ms. clm 3073, ff. 180ra-246vb. Siglo XV. Prologus: Breviarius magistri Johannis. Assiduis peticionibus me karissimi socii compendiose morborum et causas in scriptum redigere cogitis... Incipit: Quoniam longitudini in hoc opere parcere destinamus... Explicit: ...Et vos sic operamini secundum scripturam vestram opera dirigente Deo omnipotente. Deo gracias. Explicit Breviarius magistri Johannis de S[ancto] P[aulo]. Otros contenidos del códice: Bernardi de Gordonio Lectura de pronosticatione et natura morborum (ff. 1r50r); Eiusdem tractatus medicus in 4 libros divisus: de phlebotomia, de urinis, de pulsibus, de sanitatis regimine (ff. 50v-179v); Magistri Heinrici de Hassia tractatus de medicinis simplicibus particula II (ff. 247r-83v); Anonymi tractatus de regimine vitae sanitatis, aliquot versibus intermixtis: Quoniam ut ait Tullius amicitia res plurimas continet... (ff. 284r-7v); De conferentibus et nocentibus quoad omnia membra (ff. 288r-91v); Johannis de Parma practica (ff. 292r-306v) ${ }^{49}$.

${ }^{46}$ Catalogue général des manuscrits des bibliothèques publiques des départements, vol. I, Paris 1849, 416; J.-B. Hauréau, Initia operum scriptorum, vol. 1, f. 113 ${ }^{\mathrm{r}}$, col. 1, art. 7; E. Wickersheimer, Dictionnaire biographique des médecins en France au Moyen Âge. vol. III. Nouv. éd. Supplément (par D. Jacquart-G. Beaujouan), Gèneve 1979, 480; V. Rose, Egidii Corboliensis Viaticus, 103; G. H. Krömer, Johanns von Sancto Paulo, 59.

${ }^{47}$ Se trata de un códice facticio compuesto de dos manuscritos, el primero de ellos es el dedicado a la obra de Johannes de Sancto Paulo.

${ }^{48}$ W. Osler, Bibliotheca Osleriana: A Catalogue of Books Illustrating the History of Medicine and Science Collected, Arranged and Annotated, Montreal, QC, CAN 2005, 686; S. De Ricci-W. J. Wilson, Census of Medieval and Renaissance Manuscripts in the United States and Canada, vol. II, New York 1937, 2229; G. H. Krömer, Johanns von Sancto Paulo, 59; L. Thorndike-P. Kibre, A Catalogue of Incipits, 1284.

${ }^{49}$ C. Halm-G. Laubmann-G. Meyer, Catalogus codicum Latinorum Bibliothecae Regiae 
26. München, Universitätsbibliothek, $8^{\circ}$ Cod. ms. 310, ff. 1r-41v. Siglo XIIXIII: Francia. Prologus: Incipit Breviarium Johannis de Sancto Paulo... Explicit: ...ad finem usque complebimus (incompleto: transmite los libros I-IV, falta pues el libro V). Otros contenidos del códice: Incipit Modus medendi secundum Cophonem... (ff. 41v-50v); Ps. Hippocrates, Epistola ex Capsula Eburnea: Brevissima epistola Ypocratis... (ff. 50v-1r); De speciebus et herbis medicinalibus. Pro scamone... (ff. 51r-2r); De purgativis et vomitivis: De arbore laxativa... (f. 52r-v); Antidotum generale qui est ad totius corporis sanantem... (f. 52v-53r); De simplicium medicinarum virtutibus (ff. 53v-4r); Modus medendi (Renzi II 727-36): Cum inter omnia curationis genera... (ff. 55r-8r); Tractatus de aegritudinum curatione: Ordo rerum exigit sequencium... (ff. 58r-76r); De febribus: Tria sunt... (ff. 76r80r); De cyru[r]gia. Quoniam quidem de chirurgia tractaturi sumus... (ff. 80v-5v); Practica de medicinis laxativis (ff. 87r-94v); De morborum curatione et Antidota (ff. 95r-9v $)^{50}$.

27. Oxford, Bodleian Library, ms. Bodley 361 (SC 2462), ff. 203-31. Siglo XV (c. 1453-1459): Salisbury, scr. Hermannus Zurke de Greifswaldis. Prologus: Assiduis peticionibus me karissimi... Incipit: Quoniam longitudini in hoc opere parcere destinavimus... Explicit: ...opera vestra dirigente Deo omnipotenti. Amen. Gloria sit Christo, puncto sit finis in isto. Explicit opus magistri Johannis de Sancto Paulo, alme universitatis Salernitane doctoris prefamos, anno domini millesimo CCCC quinquagesimo sexto sextodecimo die mensis Octobris. Hermannus Zurhe alias de Gripessivaldis ${ }^{51}$. Otros contenidos del códice: Dietarium Stephani Arnaldi, continens tres tractatus principales (ff. 1-113); Bartholomaeus, Practica: Practica divitur in duo, in scienciam... (ff. 113-203); Tractatus de effectibus farmacorum: Parium in humano corpore multiplicitas... (ff. 331-5); Practica magistri Petri de Sancto Egidio: Cure omnium egritudinum... (ff. 335-94); Experimenta Minerve: Experimentum contra vertiginosos... (ff. 394-400); Archimatthaeus, Practica: Cum opus quodlibet suo habeat artifici... (ff. 400-24); De splene et $[\mathrm{h}]$ epate et eorum passionibus: Due vene protense sunt... (ff. 424-432); Introductorium in Practicam medicine: Cogitanti michi votum vestrum... (ff. 432-44); De dietis infirmorum secundum magistrum Petrum de Musanda: De cibis et potibus infirmorum... (ff. 444-58); Trotula maior, de passionibus mulierum [et] de causis et curis earundem: Cum auctor universitatis Deus... et Trotula minor: Ut de curis mulierum... (ff. 458-80); De ornamentis mulierum: Ut mulier suavissima appareat... (ff. 480-93); final de un 'Tractatus de pestilentia': ...salubriter convalescet (f. 493); Ricardus,

Monacensis, tomus I pars II: codices num. 2501-5250 complectens, Monachii 1871, 59; V. Rose, Egidii Corboliensis Viaticus, 103; L. Thorndike-P. Kibre, A Catalogue of Incipits, 153.

${ }^{50}$ N. Daniel, Die lateinischen mittelalterlichen Handschriften der Universitätsbibliothek München: Die Handschriften aus der Oktavreihe, Wiesbaden 1989, 154-5.

${ }^{51}$ Se refiere a Greifswald, ciudad alemana de la costa báltica. 
Compendium: Caritatis studio et breuitatis causa... (incompleto, ff. 4939); recetas médicas en inglés y latín (ff. 499-501) ${ }^{52}$.

28. Oxford, Pembroke College, ms. 13 (pars $4^{a}$ ), ff. 138r-76v. Siglo XIII ${ }^{\text {in.: }}$ Inglaterra. Prologus: Assiduis peticionibus meis karissime compendiose... Explicit: ...dirigente deo omnipotente. Otros contenidos del códice: se trata de un códice facticio formado por cuatro manuscritos del siglo XIII ensamblados en época medieval. En la cuarta parte, además del Breviarium de Johannes de Sancto Paulo, encontramos las siguientes obras: Recetas varias (ff. 176v-7v); Micrologus de causis signis et curis: Hic incipit parvus Micrologus de causis signis et curis earum de quibus agitur passionum. cum vetustati sicut moderne novitati exosa fuisset olim studiorum negociorumve prolixitas ... minora. Acutarum alia est terciana de colera rubra ... melius tinguntur quam alii (ff. 178r-200v); De signis egritudinum: (f. 201r) De signis et causis et curis egritudinum sub compendio tractaturi. quoniam teste $G$ in actionibus corporum ... de quibus aliqua utilia breuiter dicamus (f. 202) Tractatus urinarum. De urina ergo uideamus quid sit. et quot modis consideratur... (f. 205) et maxime in pleuretico et peripletico. De tracta(tu) sanorum egrorum et neutrorum: consequenter de pulsu. Hiis sufficienter et compendiose determinatis ad curarum accedamus tractatum. quare ... et ita fiat donec curetur (f. 211v) ${ }^{53}$.

29. Paris, Bibliothèque de l'Arsenal, ms. 866, ff. 34r-70v. Siglo XIII: Francia, procedente de la Bibliothèque des Carmes de la Place Maubert. Prologus: Assiduis petitionibus me karissimi compendiose morborum signa, causas et curas... Incipit: Quoniam longitudini in hoc opere parcere destinavimus... Explicit: ...vestra opera dirigente deo omnipotente, libro finito reddimus reddamus gratias Christo. Explicit liber Breviarii. Otros contenidos del códice: Parte final de un tratado sobre proporciones: [Q] uanta est distancia primi ad temperamentum, tanta est... (ff. 1r-18r); Tractatus Avicenne de medicinis cordialibus, a magistro Arnaudo de Villanova translatus: [C]reavit Deus ex concavitatibus sinistram... (ff. 18r-34r); Liber febrium Ysaac: Quoniam te, fili karissime Johannes... (ff. 73r-123v); colección de recetas médicas $(\mathrm{ff} .123 \mathrm{v}-9 \mathrm{v})^{54}$.

30. Salzburg, Universitätsbibliothek, ms. M.II.152, ff. 150ra-84va. Siglo

${ }^{52}$ F. Madan-H. H. E. Craster, A Summary Catalogue of Western Manuscripts in the Bodleian Library at Oxford which have not hitherto been catalogued in the Quarto Series, vol. 2, part 1, Oxford 1922, 378-80; M. H. Green, "A Handlist of the Latin and Vernacular Manuscripts of the so-called Trotula Texts”, Scriptorium 50/1, 1996, 159; G. H. Krömer, Johanns von Sancto Paulo, 60.

${ }^{53}$ N. R. Ker, Medieval Manuscripts in British Libraries, vol. 3, Oxford 1983, 683-4.

${ }^{54} \mathrm{H}$. Martin, Catalogue des manuscrits de la Bibliothèque de l'Arsenal, vol. 2, Paris 1886, 142; J.-B. Hauréau, Initia operum scriptorum, vol. 1, f. 113 ${ }^{\text {r }}$, col. 1, art. 7; E. Wickersheimer, Dictionnaire biographique des médecins, 480. 
XV (1467-1472): Salzburg. Prologus: Breviarius magistri Johannis. Assiduis peticionibus me karissimi socy compendiose morborum signa... Incipit: Quoniam longitudini in hoc opere parcere destinavimus... Explicit: ...secundum scripturam vestra opera dirigente Deo omnipotente. Otros contenidos del códice: Mundinus de Foro Iulio, Synonyma medicinae: Quoniam nihil carius... (ff. 1r-58v); Synonyma: Anabula assimilatur elleboro nigro... (ff. 59r-61v); Synonyma: Arthemisia vel canaparia id est matricaria... (ff. 62r-70r); Mundinus de Foro Iulio, Pondera: Pondera, gradus vel mensura... (ff. 70v-1v); De conservatione medicinarum: Aurea potest conservari... (f. 71v); Tabulae medicinarum simplicium et compositarum (ff. 73r-88r); Bernardus de Gordonio, Reginem sanitatis: Regimen excellens valde... (ff. 89r-121r); Johannes de Sancto Amando, Areolae: Sicut dicit Galenus primo... (ff. 122r-49v); Avicenna, De viribus cordis: Creavit Deus ex concavitatibus sinistram... (ff. 185r-95v); Petrus de Abano, De venenis (ff. 196r-202r); Constatinus Africanus, Liber de gradibus (ff. 202v-17v); Practica de solutivis compositis (ff. 218r-24v); Rasis, Practica puerorum (ff. 225r-7r); Practica de passione puerorum (f. 227r-v); Richardus Anglicus, Signa de distinctionibus febrium (ff. 227v-9v); Tractatus de urinis (f. 231r), de pulsu (f. 239r) et de calculo (ff. 240r-3r $)^{55}$.

31. Vaticano, Biblioteca Apostolica Vaticana, ms. Pal. lat. 1218, ff. 1ra-36vb. Siglo XIII. Prologus: Assiduis peticionibus a me karissimi compendiose ... Exequitur autem breviter signa et causas preponens et curas subiciens. Ideoque breviarium intitulavi... Incipit: Quoniam longitudini in hoc opere... (La obra se interrumpe en el capítulo De emitriteo minori del libro $\mathrm{V}$ faltándole los cinco últimos capítulos). Otros contenidos del códice: entre los folios que contienen el Breviarium se han intercalado unos versos: Dentes sic sana... (f. 1ra), el De gradibus de Urso: Quoniam in quolibet corpore elementato... (f. 15v), tres capítulos de una práctica médica: Restat tractare de passionibus sciaticis et podragricis... (f. 16r); el tratado De ponderibus Almansoris et Serapionis: (Nota: Hoc non spectat ad precedencia nec ad subsequencia sed interpositum est ne carta sit vacua) Ponderum medicinalium signa... (f. $28 \mathrm{r}-\mathrm{v}$ ) y unos experimenta: $\mathrm{Si}$ quis paraliticus loquelam amiserit... (f. 28v). Tras el texto de Johannes de Sancto Paulo se han transmitido varios capítulos de una práctica médica: ...De elefantia. elefantia quam vulgus lepram vocat... (ff. $37 \mathrm{r}-43 \mathrm{v})^{56}$.

32. Vaticano, Biblioteca A postolica Vaticana, ms. Pal. lat. 1247, ff. 1r-55v. Siglo XIII: Italia. Prologus: Incipit liber breviarii. Assiduis peticionibus me karissimi... Explicit: ...vestra opera dirigente Deo omnipotente qui vivit et regnat in secula seculorum. Amen. Explicit liber breviarii. Otros

${ }^{55}$ B. Koll, Die Handschriften des Mittelalters der Universitätsbibliothek Salzburg: Handschriftenkatalog online (http://www.ubs.ubg.ac.at/sosa/handschriften/mII152.htm).

${ }^{56}$ L. Schuba, Die medizinischen Handschriften, 219-20. 
contenidos del códice: en el f. 40v se han intercalado unos Versus de embrione. Por lo demás, el manuscrito está enteramente dedicado a la obra de Johannes de Sancto Paulo ${ }^{57}$.

33. Vaticano, Biblioteca Apostolica Vaticana, ms. Reg. lat. 1104, ff. 1ra52rb. Siglo XIV. Prologus: Assiduis peticionibus karissime conpendiose morborum signa causas et curas in scriptum redigere cogitis... Incipit: Quoniam longitudini in hoc opere parcere destinavimus... Explicit: ... in epiola oximelle et calidis utimur diureticis. Explicit breviarium. (La obra se encuentra completa). Otros contenidos del códice: Lapidarius Marbodi (glos. sive Marboti) Rodonensis: Elaus rex arabum fertur scripsisse neroni ... calore piropo. Explicit lapidarius. Explicit Marbodus (ff. 53r-62v); Incipit ovidius de mirabilibus mondi [sic]: Hic serpens ventis pernitior atque sagittis... (ff. 63r-6r); De animalibus: Picticorax bubone minor... (ff. $67 \mathrm{r}-71 \mathrm{v})^{58}$.

34. Vaticano, Biblioteca Apostolica Vaticana, ms. Urb. lat. 236, ff. 85ra90vb. Siglo XIV. Prologus: Incipit cirugia breviarii. C[apitula] de generalibus signis et causis egritudinum. Incipit: Quoniam longitudini in hoc opere peccare (sic) destinavimus ne pro nimia brevitate generemur obscuri aut medicorum vitium incurramus... Explicit: ...cassie lignee amomia a 3, ii cinnamomi 3 i. Explicit Cirurgia Abreviari (sic) philosophi in medicina Deo Gratias Amen (Se trata de un fragmento de la obra, ya que se interrumpe en el capítulo 19 del libro II titulado De dolore dentium). Otros contenidos del códice: Galeni de ingenio sanitatis: lib. III-VI Sicut operandi significationes... (ff. 1r-24v) y lib. XIII-XIV Ostendi, $k a-$ rissime frater Oceane (?) viam... (ff. 25r-32v); Cirurgia Avicenne (Canonis cum glossis libri IV fen III-IV): Iam locuti fuimus in libro primo de apostematibus... (ff. 33r-70v); Liber Constantini de oculis: Volentes oculorum infirmitates curare... (ff. 71r-80v); Rogerii Parmensis cirurgia: Quandoque sanguis discurrit... (ff. 81r-4v); Gariopontus, cirurgia Passionarii: Incipiamus de flegmone dicere... (ff. 91r-6v); Avicenne lib. IV Canonis de venenis: Qui timet quod in potu sumat venenum... (ff. $97 \mathrm{r}-118 \mathrm{v})^{59}$.

35. Venezia, Biblioteca Nazionale Marciana, ms. fon. ant. 536, ff. 44r-63v. Siglo XIV. Prologus: Incipit Breviarius Iohannis de Sancto Paulo. Assiduis peticionibus me karissimi compendiose morborum signa et causas in scriptum redigere cogitis... Incipit: Quoniam longitudini in hoc opere parcere destinavimus... Explicit: ... tam in lipparia quam in epiala oxi-

${ }^{57}$ L. Schuba, Die medizinischen Handschriften, 278.

${ }^{58}$ V. Rose, Egidii Corboliensis Viaticus, 103; J. Hamesse-S. Szyller, Repertorium initiorum manuscriptorum Latinorum Medii Aevi, vol. 1, Louvain-la-Neuve 2007, nº. 3748, 216.

${ }^{59}$ C. Stornajolo, Codices Vrbinates latini, tom. I: codices 1-500, Romae 1902, 228-30; G. H. Krömer, Johanns von Sancto Paulo, 59; L. Thorndike-P. Kibre, A Catalogue of Incipits, 1284. 
mellem et calidis utuntur diureticis. Explicit breviarius Iohannis de Sancto Paulo. Deo gratias amen. Otros contenidos del códice: De medicinis simplicibus appropiatis variis aegritudinibus: Melilotum embrocatum confert, soda, anisum... (ff. 1-28); Isaac diaetae particulares: ...I. est M. I. de Rumano (ff. 29-43) ${ }^{60}$.

${ }^{60} \mathrm{~J}$. Valentinelli, Bibliotheca manuscripta ad S. Marci Venetiarum, codices mss. Latini, tom. V, Venetiis 1872, 118-9; L. Thorndike-P. Kibre, A Catalogue of Incipits, 153. 